\title{
Comment 12.2
}

\author{
GIA NCARLO CARATTI ${ }^{1}$
}

A consensus exists that the EU's excellence in the realm of scientific research does not translate into a correspondingly high level of performance in terms of technological innovation. The perceived failure of European countries to turn scientific advances into marketable innovations is often termed the "European paradox." The innovation landscape is undergoing profound changes due to the accelerating pace of technological development, the globalization of markets, and the shortening economic life of products and processes. Hence, support frameworks for innovation and knowledge transfer are foreseen to play a highly significant role in the forthcoming EU Multiannual Financial Framework (MFF).

Although some scholars doubt the validity of this paradox, claiming that it is also a question of lower scientific quality and weak industry (Dosi et al. 2006), it is undisputable that Europe is lagging behind in terms of exploitation of its research and this is also matter of culture, for example, risk aversion, inertia, and resistance to change in universities, limited financial availability connected to an incomplete internal market, and delays in enacting legislation encouraging the exploitation of R\&D such as the Bayh-Dole Act of 1980 in the United States of America (U.S.).

Knowledge transfer offices (KTOs) play a strategic role in innovation in Europe. The adoption of standard metrics and standardized performance measurements is crucial to monitor and measure the KTOs' annual activities, and to compare and combine their results so as to get a global view of the European situation.

Many KTOs have established specialized staff and services for assessing knowledge transfer in terms of disclosed inventions, patenting, research agreements, licensing and developing, and funding spinoffs and startups. The European Commission recognizes the need for

1 The opinions expressed are those of the author only and do not necessarily reflect the position or opinion of the European Commission. 
comparable and consistent metrics across Europe regarding knowledge transfer (KT) activities in public research organizations.

A European Commission Expert Group on KT Metrics was established in 2008 in order "to identify indicators used in several existing recurrent surveys and nominate a small selection of these as core indicators, and agree on a harmonised set of definitions for them" (European Commission 2009).

Over time, the need for coherent KT metrics at the European level is still felt, and the results of the 2008 Expert Group need to be updated to take into account the specific evolving priorities, such as artificial intelligence, the Internet of Things, climate change, and the greater attention consumers are paying to the social and environmental impacts of industrial products.

In this context, a new Expert Group on Metrics for Knowledge Transfer was set up in 2019 by the European Commission's Competence Centre on Technology Transfer (CC TT), in partnership with ASTP (pan-European association for professionals involved in knowledge transfer between universities and industry) and its network of National Associations Advisory Committee (NAAC), in order to review the past work toward a key set of harmonized KT indicators that would be accepted by most in Europe. Therefore, the input provided from the authors in this chapter is both timely and useful.

The CC TT is a new service of the Joint Research Centre, which was established in 2018, and its core mission is to provide expert services to European Commission Directorates-General, regional and local authorities, and relevant stakeholders in three key areas: knowledge transfer operational support, financial instrument conception and design, and support for innovation ecosystems and clusters.

The new expert group on metrics for knowledge transfer will take into account the indicators from the European Commission report "Metrics for Knowledge Transfer from Public Research Organisations in Europe" (European Commission 2009) recent literature, current transnational and national surveys, and interviews and recommendations from national KT associations, gathered in the ASTP NAAC.

The expert group will adopt a broad concept in which knowledge transfer incorporates all functions that can lead to improved use of knowledge developed and held in the research sector for the benefit of society and its individuals. The main objectives of this expert group are the implementation of a core set of harmonized indicators, including identified risk mitigation, and the setting up of recommendations on IT 
infrastructures able to manage pan-European KT metrics data (database, security). The deliverables will be published in 2020. The expert group gathers experienced practitioners of technology and knowledge transfer with experience in $\mathrm{KT}$ activities and output measurement at a regional, national, or transnational level.

Anthony Arundel and Nordine Es-Sadki's chapter correctly points out that the indicators collected by KTOs are not capturing a significant part of knowledge transfer, which is transferred via tacit channels and, increasingly, via open science. Therefore putting emphasis only on codified knowledge may provide a distorted analysis of the ability of a research organization to transfer its knowledge. The authors propose additional indicators using specific surveys of academics or firms to complement the data from KTOs. In my view, this is an interesting proposal from a theoretical point of view but it is also very challenging to put into practice. Besides the high organizational costs of consulting a large number of academics and industries (and their survey fatigue), ASTP already finds it challenging to consult the existing KTOs in Europe, with the result that their statistical data have a skewed geographic coverage. One of the reasons is that the current set of indicators is probably too large, and many small KTOs cannot regularly monitor all of them. One of our recommendations to the experts undertaking the review of the KT metrics would therefore be to develop as simple as possible a system of indicators and to specify other potentially important factors, in most cases nonmeasurable, that contribute to the success of the knowledge transfer process. Some expert readers will be aware of "Goodhart's Law": "when a measure becomes a target, it ceases to be a good measure."

\section{References}

Dosi, G., P. Llerena, and M. Sylos Labini (2006). The Relationships Between Science, Technologies and Their Industrial Exploitation: An Illustration Through the Myths and Realities of the So-Called "European Paradox." Amsterdam: Elsevier.

European Commission (2009). Metrics for Knowledge Transfer from Public Research Organisations in Europe. Report from the European Commission's Expert Group on Knowledge Transfer Metrics. ec.europa.eu/invest-in-research /pdf/download_en/knowledge_transfer_web.pdf. 